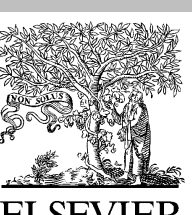

Available online at www.sciencedirect.com

S CIENCE

JOURNAL OF

\title{
Analytical modeling of codes with arbitrary data-dependent conditional structures
}

Universidade da Coruña, Depto. de Electrónica e Sistemas, Facultade de Informática, Campus de Elviña s/n, 15071 A Coruña, Spain

Received 20 May 2004; received in revised form 28 January 2005; accepted 24 April 2005

8 Abstract

Several analytical models that predict the memory hierarchy behavior of codes with regular access patterns have been developed. These models help understand this behavior and they can be used successfully to guide compilers in the application of locality-related optimizations requiring small computing times. Still, these models suffer from many limitations. The most important of them is their restricted scope of applicability, since real codes exhibit many access patterns they cannot model. The most common source of such kind of accesses is the presence of irregular access patterns because of the presence of either data-dependent conditionals or indirections in the code. This paper extends the probabilistic miss equations (PME) model to be able to cope with codes that include data-dependent conditional structures too. This approach is systematic enough to enable the automatic implementation of the extended model in a compiler framework. Validations show a good degree of accuracy in the predictions despite the irregularity of the access patterns. This opens the possibility of using our model to guide compiler optimizations for this kind of codes. (C) 2005 Elsevier B.V. All rights reserved.

\section{Introduction}

There has been a growing interest in the study and understanding of the behavior of the memory hierarchies in the past years. The reason is the essential role they play in the performance of modern

\footnotetext{
This work was supported in part by the Ministry of Education and Science of Spain under contracts TIC2001-3694-C02-02 and TIN2004-07797-C02-02, and by the Xunta de Galicia under contract PGIDIT03TIC10502PR.

* Corresponding author. Tel.: +34 981 167000x1219; fax: +34 981167160.

E-mail addresses: dcanosa@udc.es (D. Andrade), basilio@udc.es (B.B. Fraguela), doallo@udc.es (R. Doallo).
}

computers, mainly because of the increasing differ- 27 ence between main memory and processor speeds. 28 One of the most effective ways to reduce the impact 29 of this difference is the usage of memory hierarchies 30 with one or, more typically, several level of caches. 31

The first approach to study the behavior of these 32 systems was the usage of trace-driven simulations 33 [1]. This approach, while very accurate, has many 34 drawbacks: difficulty to store the traces, large com- 35 puting times, and lack of an explanation for the 36 behavior observed in many cases. The first two 37 problems can be overcome by the usage of hardware 38 counters [2], but they still offer no explanations 39 about the behavior observed and they are restricted 40 
41 to the platforms in which they are available.

42 Besides, none of those approaches is suitable to

43 guide the optimization process of a compiler. This

44 way, a number of analytical models have appeared

45 that try to address these issues [3-7].

46 Analytical models suffer typically from two kinds

47 of problems: a certain lack of accuracy and a limited

48 scope of applicability, either because of the limited

49 number of code structures that can model or

50 because of a restriction to model a given kind of

51 hardware. Some of the most recent models have

52 achieved very good degrees of accuracy in their pre-

53 dictions, and they are general enough to consider

54 both the direct-mapped and set-associative caches

55 with LRU replacement that are found nowadays

56 in almost every computer. Still, they continue to

57 restrict their applicability to codes that must exhibit

58 regular access patterns. Unfortunately, most real

59 codes comprise either indirections or portions of

60 code whose execution depends on conditions com-

61 puted at run-time. These structures break the regu-

62 larity of the accesses and, as a result, they are

63 beyond the scope of these models.

64 In this paper we extend one of these models, the

65 probabilistic miss equations (PME) model [7], to

66 enable it to analyze automatically codes that include

67 data-dependent conditional structures. We will

68 consider codes with any kind and number of condi-

69 tional sentences, even with references controlled by

70 several nested conditionals, and nested in any arbi-

71 trary way. Only two restrictions are set on the

72 conditions. The first one is that their verification

73 must follow an uniform distribution, although each

74 condition may have a different probability of being

75 fulfilled. The second one is that the conditions must

76 be independent, this is, the probability a given con-

77 dition is fulfilled is not influenced by the fact any

78 other condition(s) is/are fulfilled or not. These

79 restrictions ease the mathematical treatment of the

80 problem in this first attempt to model automatically

81 codes with irregular access patterns, while allowing

82 to represent the most important modeling problems

83 derived from such irregularities. Still, we acknowl-

84 edge these conditions do not hold in most real

85 codes. This way, we are currently working in the

86 modeling of conditions that are fulfilled with non-

87 uniform distributions.

88 The PME model, which we describe in detail in

89 Section 2, builds a separate expression for each ref-

90 erence and each loop that encloses it, that estimates

91 the number of misses generated by the reference

92 during the execution of that loop. Its equations are probabilistic because the number of misses is 93 estimated as the product of the estimated number 94 of accesses by the estimated probability each one 95 of those accesses generates a miss. Such probability 96 is derived from the footprint on the cache of the dif- 97 ferent regions accessed between two consecutive 98 accesses to the same line by the reference that is 99 being analyzed. This way, the original PME model 100 in [7] only used probabilities to describe the probability an access resulted in a miss, while the number of accesses and the shape of the footprints was fixed. Our extension also uses probabilities to estimate the number of accesses, and to estimate the footprint of the regions that can preclude a reuse in an access. The reason is that references affected by data-dependent conditionals only take place with a given probability. As a result, a new strategy to generate probabilistic miss equations has been developed to deal with these codes.

Notice that the PME model provides more information than other analytical models of the memory because it generates an individual equation for each reference and nesting level, and the miss probabilities are computed adding the contributions of the accesses of the different references found within the reuse distance. This way, a very detailed individual analysis for every reference and how it influences the behavior of other references is provided.

This paper is structured as follows: The following section provides an introduction to the PME model extensively described in [7]. Then, Section 3 describes the scope of application of the new extension and its formulation. Section 4 is devoted to the validation of the extended model. A brief review of the related work is presented in Section 5, followed by our conclusions and a discussion on the future work in Section 6.

\section{Probabilistic miss equations (PME) model}

As mentioned in the previous section, the PME model is originally oriented to the modeling of codes with regular access patterns. The model considers caches of an arbitrary size, line size and associativity whose replacement policy is LRU. It supports both perfectly and imperfectly nested loops with a fixed number of iterations. The model allows several references per data structure and loop, and it requires the indexing functions for the different dimensions of the references to be affine functions of the enclosing loops index variables, which is the most common situation. The model
101

102

103

104

105

106

107

108

109

110

111

112

113

114

115

116

117

118

119

120

121

122

123

124

125

126

127

128 
143 can also take into account the probability of hit due

144 to the reuse of cache lines in different loop nests,

145 which enables it to model complete codes. Still,

146 the inter-nest reuse modeling accuracy is subject to

147 the fulfillment of certain conditions.

148 The estimation of the number of misses gener-

149 ated by the execution of a given code in a certain

150 cache is made separately for each reference in this

151 model. In fact, the model generates a separate equa-

152 tion for each loop and for each reference that esti-

153 mates the number of misses it generates in that

154 loop. This is modular and it allows the user to know

155 which are the hot spots and references in the code.

156 The model classifies misses in two categories. Com-

157 pulsory misses are those that take place the very first

158 time a line is referenced in the code. Interference

159 misses are attempts to reuse a line that fail because

160 the line was evicted from the cache since its previous

161 access. The distinction is reflected in the way the

162 PMEs are built, as each kind of misses is estimated

163 separately. The references that can give place to a

164 reuse are also classified in their turn according to

165 their reuse distance, this is, the portion of code exe-

166 cuted since the latest access to the line they try to

167 reuse. The reason is that different reuse distances

168 have associated a different probability of resulting

169 in a miss. The number and type of the different

170 accesses is estimated from the indexing functions

171 of the references and the sizes of the loops.

172 The probabilistic nature of the PME model

173 comes into play when the interference misses are

174 estimated. They are calculated separately for each

175 potential reuse distance, as the product of the

176 number of accesses that could enjoy a potential

177 reuse of a line in the cache with that distance, by

178 the probability each access really results in a miss.

179 The probability is estimated from the cache foot-

180 print of those regions that have been accessed since

181 the latest reference to the line, this is, during the

182 considered reuse distance.

183 We will now describe the strategy to represent

184 these footprints and estimate the corresponding

185 miss probabilities and how PMEs are built for refer- ences that are not subject to conditional accesses, 186 this is, those considered in [7].

\subsection{Miss probability calculation}

The PME model measures reuse distances in terms of loop iterations. Fig. 1 shows the steps the PME model follows to derive the miss probability associated to a given reuse distance. We will now comment them in turn.

190

191

192

193

\subsubsection{Access pattern identification}

In the first step, the access pattern followed by the references involved in a reuse distance is extracted from their indexing functions and the shape of the loops that enclose them. This task is eased due to the usage of affine indexing functions in the references considered by the model. The access patterns can be described by means of the memory regions they reference, using for example notations like the Access Region Descriptors [8]. Nevertheless, the PME model represents access patterns as functions whose output is the footprint of the access on the cache. The model associates a different function to each typical class of access pattern found in the codes analyzed (sequential access, access to regions separated by a constant stride, etc.). The function arguments complete the description of the access pattern. For example, the only argument required to characterize a sequential access is the number of words accessed.

\subsubsection{Cache impact quantification}

The second step evaluates the access pattern 215 functions to obtain their associated cache foot- 216 prints. These footprints are represented in the 217 PME model by what we call area vectors. An area 218 vector $V$ consists of $K+1$ probabilities $V_{0} V_{1} \ldots V_{K}, 219$ where $K$ is the degree of associativity of the cache 220 whose behavior is analyzed. This representation is 221 designed to be very convenient for the calculation 222 of the impact of the corresponding accesses on the 223 miss probability when trying to reuse lines from

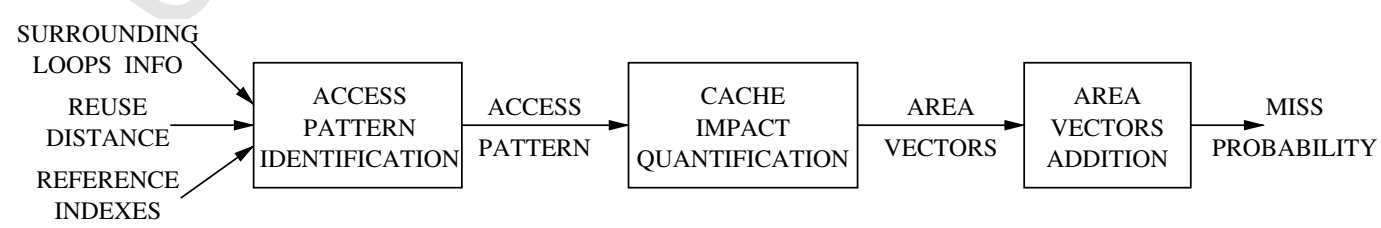

Fig. 1. Procedure for estimating miss probabilities from the code. 
225 other access patterns or even from the access that is 226 being considered. In fact, two kinds of area vectors 227 are distinguished:

- Cross-interference area vectors represent the impact on the cache of the considered access pattern as viewed by lines not involved in the access. In these vectors, the first component, $V_{0}$, is the probability that a set in the cache has received $K$ or more lines accessed by the pattern. One can think of this probability also as the ratio of cache sets that have received $K$ or more lines during the access. Then, $V_{1}$ is the probability a cache set has received exactly $K-1$ lines; $V_{2}$ is the probability a cache set has received exactly $K-2$ lines; and so on. In general, except for $i=0, V_{i}$ is the probability a given set has received exactly $K-i$ lines due to the access.

- Self-interference area vectors represent the impact of the footprint on the probability of reuse for the lines it involves. In these vectors, $V_{0}$ is the probability that a line of the footprint is competing in its cache set with other $K$ or more lines of the footprint. For $i>0, V_{i}$ is the probability a line of the footprint shares its cache set with other $K-i$ lines of the access.

Example. Let us consider a simple cache footprint in a 2-way associative cache with eight sets such that 7 of the 8 sets have received two lines, and the other set has only received one line. The cross-interference area vector $V_{\text {cross }}$ for this footprint is $(7 / 8,1 / 8,0)$, since 7 out of the 8 sets have received two or more lines from the access; only one set received a single line, and no sets received zero lines. These ratios are conversely the probabilities a randomly chosen set has two or more, one, or zero lines in it, respectively.

The self-interference area vector $V_{\text {self }}$ for this footprint is $(0,14 / 15,1 / 15)$. Its first component indicates that none of the lines involved in the access has to compete for its cache set with other two or more other lines from the access pattern. The second component is the ratio of lines of the footprint that share their cache set with exactly one line (14 out of 15). Finally, as the third component points out, only one of the fifteen lines of the footprint does not share its set with any other line of the footprint. These ratios are conversely the probabilities a randomly chosen line of the footprint has to compete in its set with two or more, one, or no lines, respectively.
Area vectors are derived for each access pattern 276 either analytically or by simulation or following a 277 hybrid approach. The method to estimate the area 278 vectors associated to the most commonly found 279 access patterns has been described in [7]. Section 280 3.1 describes the estimation of the area vector for 281 two new access patterns not previously considered.

\subsubsection{Area vectors addition}

283

Interference probabilities are directly obtained 284 from area vectors because in a $K$-way associative 285 cache, the probability of missing in the cache when 286 trying to reuse a line corresponds to the probability 287 that $K$ (or more) different lines, mapped to the cache 288 set associated with that line, have been referenced 289 since its previous access. This is exactly the first 290 component of any area vector. The other compo- 291 nents are also required because several data struc- 292 tures may be accessed during a given reuse 293 distance. The PME model estimates the area vector 294 for the accesses to each structure separately and 295 then adds them to calculate the global area vector 296 in the third step of the process depicted in Fig. 1, 297 the Area Vectors Addition. This way, components 298 not in the first position of their corresponding area 299 vectors may be combined to increase the probability 300 that in the global footprint there are $K$ or more lines 301 mapped to a cache set. The addition of area vectors, 302 whose operand is $U$, is described in detail in [7].

\subsection{Condition independent PMEs}

304

The PME model numbers the loops in a nest 305 from the outermost one, zero, to the innermost 306 one, $Z$; and it analyzes the behavior of the refer- 307 ences beginning in the innermost loop that contains 308 them and proceeding outwards. This way, the model 309 generates an estimator $F_{i}(R, \operatorname{RegIn})$ of the number 310 of misses generated by each reference $R$ during the 311 execution of each enclosing loop at nesting level $i . \quad 312$ This PME depends on RegIn, the footprints gener- 313 ated by regions accessed in outer loops that may 314 interfere with the reuse of the footprint of $R$ in 315 loop $i$.

Every estimator is a summatory. The first term corresponds to the accesses that cannot enjoy reuse in the considered loop, so it is associated to the misses that are compulsory from the point of view of the loop. The miss probability for these accesses depends on RegIn, the footprint due to accesses in outer loops. The remaining terms correspond to the accesses that can enjoy reuse, there being one

7
8
9
0
1

2

3


325 term for each different potential reuse distance.

326 Every term is a product of the estimated number 327 of accesses that reuse cache lines with a given reuse 328 distance multiplied by the miss probability associ329 ated to that distance.

330 A description on how to derive PMEs both for 331 references that can and cannot reuse lines accessed 332 by other references is found in [7]. In order to make 333 this paper more self-contained and help understand 334 our extension in Section 3, we will explain here the 335 construction of PMEs for references that carry no 336 reuse with other references in its loop nest. These 337 PMEs are built as

$$
\begin{aligned}
F_{i}(R, \operatorname{RegIn})= & L_{R i} F_{i+1}(R, \operatorname{RegIn}) \\
& +\left(N_{i}-L_{R i}\right) F_{i+1}\left(R, \operatorname{Reg}_{i}(R, 1)\right),
\end{aligned}
$$

341 where $N_{i}$ is the number of iterations of the loop at 342 nesting level $i, L_{R i}$ is the number of iterations in 343 which $R$ cannot reuse lines in this loop, $F_{i+1}(R, \mathrm{Re}-$ $344 \mathrm{gIn})$ is the PME for the same reference $R$ in the 345 immediately inner loop and $\operatorname{Reg}_{i}(R, n)$ are the re346 gions accessed during $n$ iterations of the loop $i$ that 347 may interfere with the accesses of $R$. The formula 348 reflects that the miss probability for the $L_{R i}$ loop 349 iterations in which there can be no reuse in this 350 loop, depends on the accesses in the outer loops (gi351 ven by RegIn), while the miss probability for the 352 accesses in the remaining iterations is a function of 353 the regions accessed during the portion of the pro354 gram executed between those reuses, which is one 355 iteration of this loop. Notice how the calculation 356 for the PME in level $i$ provides the RegIn argument 357 for $F_{i+1}$, which estimates the behavior of $R$ during 358 the execution of the immediate inner loop.

359 Two special cases must be considered when eval360 uating the PMEs:
- In the innermost loop $F_{i+1}(R, \operatorname{RegIn})=A V_{0}(\mathrm{Re}-$ gIn), this is, the first element of the area vector associated to the region RegIn. The reason is that the estimator is associated here to a single access in a single iteration of this innermost loop.

- When the outermost loop is reached, the input region for $F_{0}(R, \operatorname{RegIn})$, which estimates the total number of misses generated by $R$ in the nest, is RegIn ${ }_{\text {total }}$, an imaginary region that covers the whole cache and that generates a miss probability one. The reason is that the PMEs propagate this region as RegIn for those accesses that carry no reuse at all in the nest and which, as a result, are compulsory misses for the nest.
Since the indices of the references are affine functions of the enclosing loop variables, the accesses of every reference $R$ have a constant stride $S_{R i}$ associated to the loop $i$. Consequently, the number of different lines that are accessed in $N_{i}$ iterations with stride $S_{R i}$, can be calculated as

$L_{R i}=1+\left\lfloor\frac{N_{i}-1}{\max \left\{L_{\mathrm{s}} / S_{R i}, 1\right\}}\right\rfloor$,

where $L_{\mathrm{s}}$ is the number of array elements a cache line holds. This $L_{R i}$ value corresponds also to the number of iterations in which the accesses of $R$ cannot reuse lines brought to the cache by previous accesses in this loop. The remaining $N_{i}-L_{R i}$ iterations can exploit either spatial or temporal locality, with a reuse distance of a single iteration of the considered loop.

\section{Modeling of condition dependent references}

The modeling strategy described in the preceding section is valid for codes without conditional sentences, which is the scope of application of all the previous works in the bibliography, as we will see in Section 5. Only Vera and Xue [9] has considered codes with conditional sentences, but it is restricted to conditions on the loop indices, which are completely predictable and analyzable off-line and which tend to follow quite regular patterns. In practice, many codes include data-dependent conditionals whose outcome depends on computations made at run-time, and where the pattern of the condition is highly irregular. As a result, the references affected by those conditions exhibit very irregular access patterns that no model has managed to analyze following a modular and systematic approach. This is the main contribution of our work.

The scope of application of our model is shown in Fig. 2. We now consider any number of arbitrarily nested conditional statements, with an arbitrary number of atomic conditions that involve any number of data elements. The figure only shows one data element per condition for simplicity. The IF structures condition the execution of isolated references or complete loops or nests. The restrictions in the PME model of constant number of loop iterations and affine indexing continue to hold. Also, our current systematic strategy to model irregular access patterns requires the conditions in the code to follow an uniform distribution and to be independent. This latter restriction means that the 


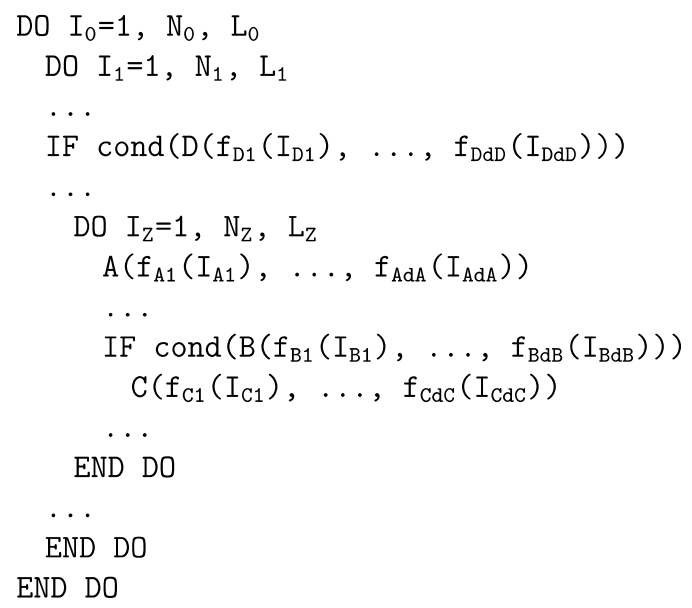

Fig. 2. Loop nest with data-dependent conditions.

424 probability that a given condition is fulfilled or not 425 does not depend on the verification of other condi426 tions in the code. We expect to relax these restric427 tions in future works. The different conditions 428 may be fulfilled with different probabilities each.

429 Two kinds of extensions are required to consider 430 irregular accesses. One is the identification of new 431 access patterns that give place to footprints not con432 sidered by the original PME model, and for which 433 methods must be developed in order to estimate 434 their corresponding area vectors. The other one is 435 the consideration of a new kind of PMEs in which 436 reuses take place only with a given probability, 437 and whose reuse distance varies depending on the 438 behavior of the conditional sentences found in the 439 nest. We will now consider in turn these two issues.

\subsection{Irregular access patterns}

The two access patterns usually found in codes with regular access patterns are the sequential access and the access to groups of consecutive elements of the same size that are separated by a constant stride. Their irregular counterparts, when uniform probabilities of access are considered, are described in a similar way, with the important difference that now each one of the elements involved in the pattern is accessed with a given probability $p$ that is the same one for every element. The modeling of these new access patterns, which we detail below, depends on the cache parameters. A cache is defined by its total size $C_{\mathrm{s}}$, its line size $L_{\mathrm{s}}$, and its associativity $K$. For simplicity, both $C_{\mathrm{s}}$ and $L_{\mathrm{s}}$ are measured in elements or words of the access we are considering. Two derived parameters that help simplify some expressions are the number of sets in the cache, 457 $N_{K}=C_{\mathrm{s}} /\left(K L_{\mathrm{s}}\right)$, and $C_{\mathrm{sk}}=C_{\mathrm{s}} / K$, the cache size 458 devoted to each level of associativity.

\subsubsection{Sequential access with uniform probability}

We denote as $S_{\mathrm{sp}}(n, p)$ the cross-interference area vector associated to an access to $n$ consecutive elements in which each one of them has a probability $p$ of being referenced. The $K+1$ elements of this vector are calculated as

$S_{\mathrm{sp}_{i}}(n, p)=P(X=K-i) \quad m<i \leqslant K$,

$S_{\mathrm{sp}_{m}}(n, p)=P(X \geqslant K-m)$,

$S_{\mathrm{sp}_{i}}(n, p)=0 \quad 0 \leqslant i<m$,

where $X \in B\left(n / C_{\mathrm{sk}}, 1-(1-p)^{L_{\mathrm{s}}}\right)$, being $B(n, p)$ the binomial distribution ${ }^{1}$ and $m=\max \{0, K-\lceil n]$ $\left.\left.C_{\mathrm{sk}}\right\rceil\right\}$. The formula is based on the fact that, on average, there are $n / C_{\text {sk }}$ lines of the footprint associated to each cache set. Since this is a consecutive memory region, the maximum number of lines a cache set can receive is $\left\lceil n / C_{\mathrm{sk}}\right\rceil$, so the area vector elements $S_{\mathrm{sp}_{i}}(n, p)$ for $0 \leqslant i<m$ must be zero. Also, because of the uniform distribution of the accesses, we know that the number of cache lines per set belongs to a binomial $B\left(n / C_{\text {sk }}, 1-(1-p)^{L_{\mathrm{s}}}\right)$. The probability of access per line of this binomial is easy to calculate, as since each individual element in a cache line has a probability $p$ of begin accessed, and a line holds $L_{\mathrm{s}}$ elements, then the probability that at least one of the elements of the line receives a reference is $1-(1-p)^{L_{\mathrm{s}}}$. Since position $i, i>0$, in the area vector represents the ratio of sets that receive $K-i$ lines in the access, its value will be the probability the variable associated to this binomial takes the value $K-i$. The lowest element in the area vector with non-zero probability, $m$, is the probability the number of lines accessed is $K-m$ or more.

\subsubsection{Access to groups of elements separated by a constant stride with uniform probability}

We denote as $S_{\mathrm{rp}}\left(N_{\mathrm{r}}, T_{\mathrm{r}}, L_{\mathrm{r}}, p\right)$ the cross-interference area vector associated to an access to $N_{\mathrm{r}}$ regions of $T_{\mathrm{r}}$ consecutive elements each and separated by a constant stride of $L_{\mathrm{r}}$ elements, in which each individual element has a probability $p$ of being

468 469 470 471 472 473 474 475 476 477 478 479 480 481 482 483 484 485 486 487 488 489 490 491 493 494 495 496 497 498 
499 referenced. This area vector is calculated in two 500 phases:

$$
\begin{aligned}
N(j, i)= & C V\left(\operatorname{pos}\left(j L_{\mathrm{s}}-T_{\mathrm{r}}+i\right)\right) \\
& +C V\left(p o s\left(j L_{\mathrm{s}}+L_{\mathrm{s}}-i\right)\right)
\end{aligned}
$$

531 since only the regions that begin exactly $T_{\mathrm{r}}-i$ posi-

540 since the regions beginning in the first $L_{\mathrm{s}}-T_{\mathrm{r}}+1$ 541 positions of the set will have one line in which $T_{\mathrm{r}}$ 542 of its elements may be accessed, and given that
$T_{\mathrm{r}}<L_{\mathrm{s}}$, it is impossible that there are regions with lines where more than $T_{\mathrm{r}}$ elements may be accessed.

Finally, if $T_{\mathrm{r}} \geqslant L_{\mathrm{s}}$, all the $N(j, i)$ but $N\left(j, L_{\mathrm{s}}\right)$ have been calculated. The value for the latter is calculated as

$N\left(j, L_{\mathrm{s}}\right)=\sum_{t=L_{\mathrm{s}}}^{T_{\mathrm{r}}} C V\left(p o s\left(j L_{\mathrm{s}}-T_{\mathrm{r}}+t\right)\right)$

because any region that begins either in the first position of the set or in the $T_{\mathrm{r}}-L_{\mathrm{s}}-1$ immediately preceding positions will have one line mapped to the considered set $j$ in which all of its elements may be affected by the access pattern.

3.1.2.2. Weighting the accesses probabilities. In the previous phase we have estimated the footprint of this access pattern without taking into account the probability that each element in the footprint is really referenced. Let us remember that the footprint is represented by the values $N(j, i)$, which are the number of lines mapped to set $j$ that contain $i$ words affected by the access pattern. Since the access to each element happens only with probability $p$, this is an upper bound of the real number of lines that are accessed. This way, the purpose of this phase is to estimate how many lines are really accessed taking into account that the probability of access to each element in the region is $p$.

Our strategy to estimate the total area vector for this access pattern is to calculate the area vector for each set $j$ independently and to average them. The area vector for each single set $j, S_{j}$, represents the distribution of probability that the access generated references to $l$ different lines mapped to this set for $0 \leqslant l<K$ in the positions $S_{j(K-l)}$ of the vector, or to $K$ or more different lines, in the position $S_{j 0}$. This distribution of probability is calculated from $L_{\mathrm{s}}$ binomial variables, $X_{j i}, 1 \leqslant i \leqslant L_{\mathrm{s}}$, where $X_{j i}$ is the number of lines that are really accessed out of the $N(j, i)$ ones that are mapped to set $j$ and which contain exactly $i$ positions that can be referenced by the access pattern analyzed. This way, $X_{j i} \in B(N(j, i)$, $\left.1-(1-p)^{i}\right)$, where $B(n, p)$ stands for the binomial distribution. The probability of the binomial is given by the fact that if in a given line only $i$ positions may be subject to access, and the access to each position only happens with probability $p$, then the probability the line has really been accessed is $1-(1-p)^{i}$. As a result, if we define $X_{j}=$ $\sum_{i=1}^{L_{\mathrm{s}}} X_{j i}$, then the area vector for the set $j$ can be estimated as $S_{j(K-l)}=P\left(X_{j}=l\right), 0 \leqslant l<K$ and $S_{j 0}=$ $P\left(X_{j} \geqslant K\right)$. 


\subsection{Condition dependent PMEs}

In order to consider the probabilities that the different conditional statements that may affect a given reference $R$ in its nest hold, we extend the PME that estimates the behavior of a reference $R$ in a loop $i$ with a new argument $\vec{p}$. This vector contains in position $j$ the probability $p_{j}$ that the (possible) conditionals that guard the execution of the reference $R$ in nesting level $j$ are verified. If a given loop contains no conditional structures, then $p_{j}=1$, which means the execution in this level is unconditional. When there are several nested IF statements in the same nesting level, $p_{j}$ is the product of the probabilities of holding their respective conditions.

We have found that $F_{i}(R, \operatorname{RegIn}, \vec{p})$ may take two different forms when considering codes with datadependent conditional statements. If the reference is not affected by any conditional sentence or if the variable that indexes loop $i$ does not index any of the references found in the condition(s) of the conditional(s) sentence(s) that affect the execution of $R$, then the PME takes the form described in Section 2.2. This kind of PME disregards its input $\vec{p}$, which is not used in the computations. But if this is not the case, this is, if the variable of the loop is used in the indexing of a data array involved in a conditional that controls the execution of the reference $R$ that is being studied, then a new kind of PME must be used. From now on we will distinguish both kinds of PMEs by calling the former ones Condition Independent PMEs and these new ones Condition Dependent PMEs.

Just as we did in Section 2.2, we will now describe the construction of Condition Dependent PMEs for references that carry no reuse with other references. We will do it in two steps. First, we will develop the general form of a Condition Dependent PME. This PME is based on the probability that the reference that is being analyzed actually accesses each one of the lines of the set that the reference can potentially access during one iteration of the loop $i$ we are considering. In a second step, an algorithm to derive this probability will be presented.

\subsubsection{General form of a condition dependent PME}

A PME must be built for each loop $i$ enclosing a reference $R$. The PME is basically a summatory where each term is the product of the number of accesses that have a given reuse distance, multiplied by the PME for the lower level when the input footprint corresponds to that reuse distance. When ref- erence $R$ is affected by data-dependent conditionals, this is, when one or more IF structures that depend on data control the reference, the reuse distances are not fixed. Depending on the pattern of verification of the conditions that control the execution of the reference, its accesses may try to reuse lines with very different distances. These reuse distances will have different probabilities of happening, depending on the distribution of probability of the verification of the conditionals that control the execution of the reference. This way, the PMEs for this kind of references will use probabilities not only to represent the miss probability for a given reuse distance, as those in Section 2.2 did, but also to estimate how many accesses take place with each possible reuse distance. Notice that PMEs measure the reuse distance in terms of iterations of the loop they are associated to, and the unit of reuse in a cache is the line. As a result, the base probability to weight the different reuse distances must be the probability that the reference that is being analyzed accesses one of the lines it may potentially access during each iteration of the loop $i$ that is being considered. In general, when the conditionals do not follow an uniform distribution, a set of different probabilities for different iterations and/or lines must be used. As the scope of this analysis is restricted to conditionals that follow an uniform distribution, in this work this probability is a single parameter, $P_{i}(R, \vec{p})$, that has the same value for every iteration of the loop $i$ and for every line that $R$ may access. This way, the condition dependent PME for loop $i$ and reference $R$ has the form

$$
F_{i}(R, \operatorname{RegIn}, \vec{p})=p_{i} L_{R i} \sum_{j=1}^{G_{R i}} \mathrm{WMR}_{i}(R, \operatorname{RegIn}, j, \vec{p}),
$$

where $L_{R i}$ is the number of iterations in which new different lines would be accessed by reference $R$ due to the stride in loop $i$ if it were not subject to conditional execution, and $p_{i}$ is the probability the conditional sentences that control the execution of $R$ in this loop level are true. The product of these two terms gives the average number of iterations in which $R$ accesses different lines due to its stride for this loop. This number of iterations must be multiplied by the PME for the immediately lower level evaluated with the appropriate reuse distance area vector, which is what the term $\mathrm{WMR}_{i}$ stands for, a weighted number of misses for a reference in level $i$. As stated before, because of the control by data- 
693 dependent conditionals, a range of different reuse 694 distances with different probabilities may take place. 695 This range has an average upper bound $G_{R i}$, the 696 number of iterations that can potentially reuse the 697 lines accessed in the $L_{R i}$ iterations that give place 698 to accesses to new lines. The product of both terms 699 must be equal to the number of iterations of the 700 701 702 703

704 705 loop, thus $G_{R i}=N_{i} / L_{R i}$.

Let us now develop the value of $\mathrm{WMR}_{i}$ $(R, \operatorname{RegIn}, j, \vec{p})$, the weighted number of misses generated by reference $R$ in loop $i$ when RegIn is the region accessed since the last access to any of the lines affected by the reference of $R$ before loop $i$ begins its execution, and the line is used in the $j$ th possible iteration in which the line could be accessed. This function is computed as

$$
\begin{aligned}
& \mathrm{WMR}_{i}(\operatorname{RegIn}, j, \vec{p}) \\
&= \overline{P_{i}(R, \vec{p})}{ }^{j-1} F_{i+1}\left(R, \operatorname{RegIn} \cup \operatorname{Reg}_{i}(R, j-1), \vec{p}\right) \\
&+\sum_{k=1}^{j-1} P_{i}(R, \vec{p}){\overline{P_{i}(R, \vec{p})}}^{k-1} F_{i+1}\left(R, \operatorname{Reg}_{i}(R, k), \vec{p}\right),
\end{aligned}
$$

712 where $P_{i}(R, \vec{p})$, the probability that $R$ accesses dur713 ing one iteration of loop $i$ one of the lines that be714 long to its potential access pattern, is used to 715 weight the probabilities that the different reuse dis716 tances take place. In this equation $\bar{p}$ stands for $7171-p$, this is, the converse probability of $p$. Let us 718 remember that $\operatorname{Reg}_{i}(R, n)$ stands for the regions ac719 cessed during $n$ iterations of the loop $i$ that may 720 interfere with the accesses of $R$. The first term in 721 Eq. (4) considers the case that the line has not been 722 accessed during any of the previous $j-1$ iterations. 723 In this case, the RegIn region that could generate 724 interference with the new access to the line when 725 the execution of the loop begins, must be added to 726 the regions accessed during these $j-1$ previous iter727 ations of the loop in order to estimate the complete 728 interference region. The second term weights the 729 probability that the last access took place in each 730 of the $j-1$ previous iterations of the considered 731 loop. level, but also in the inner ones, so its calculation takes into account all the loops from the $i$ th down to the one containing the reference. If fact, this probability is calculated recursively in the following way:

- If $i$ is the innermost loop containing $R$, then

$P_{i}(R, \vec{p})= \begin{cases}1 \quad \text { if the accesses of } R \text { are consecutive } \\ \quad \text { with respect to loop } i \\ p_{i} \quad \text { otherwise }\end{cases}$

where a consecutive access with respect to a given loop implies that the accesses that take place in consecutive iterations of the loop do reference consecutive memory positions. The condition for this to happen even when the accesses of $R$ depend on an IF statement is that the index for the first dimension of $R$ only makes (sequential) progress within the same IF statement that controls $R$. As an example, this is what happens with references $B(p \circ s B)$ and $j B(p \circ s B)$ in the innermost loop of the CRS code (Fig. 4) that we use in Section 4 to validate our model: their index posB only advances when these references take place; thus consecutive accesses affect consecutive memory positions, even if the references are controlled by a condition.

- If $i$ is not the innermost loop containing $R$, then

$$
\begin{aligned}
& P_{i}(R, \vec{p}) \\
& = \begin{cases}p_{i} P_{i+1}(R, \vec{p}) & \text { if the index of loop } i+1 \text { is } \\
& \text { not used in the references found in } \\
& \text { conditions that control } R, \\
p_{i} \overline{\bar{P}_{i+1}(R, \vec{p})} G_{R_{i+1}} & \text { otherwise, }\end{cases}
\end{aligned}
$$

where we must remember that $\bar{p}=1-p$ and that $p_{i} 765$ is the product of all the probabilities associated to 766 the conditional sentences affecting $R$ in nesting level $i .767$

\section{Validation}

Our validation of the model is based on the comparison of its cache miss predictions with the result of trace-driven simulations. We have used three simple kernels shown in Figs. 3-5. The first code is a synthetic kernel with a conditional sentence that control the access to a data structure C. Then, Fig. 4 implements the storage of a matrix in CRS format (Compressed Row Storage), which is widely used to store sparse matrices in a compressed form. The code has two nested loops and
747 748 749 750 751 752 753 754 755 756 757 758 759 760 761 762 


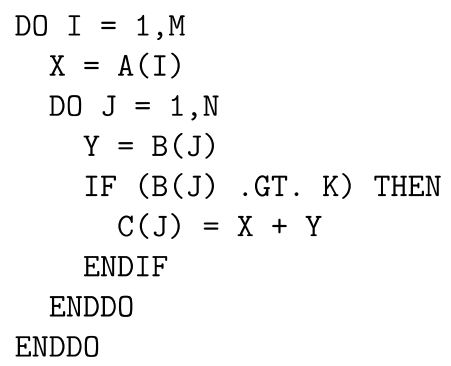

Fig. 3. Synthetic kernel code.

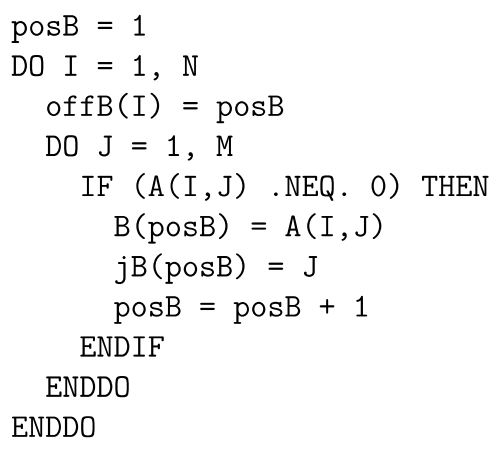

Fig. 4. CRS storage algorithm.

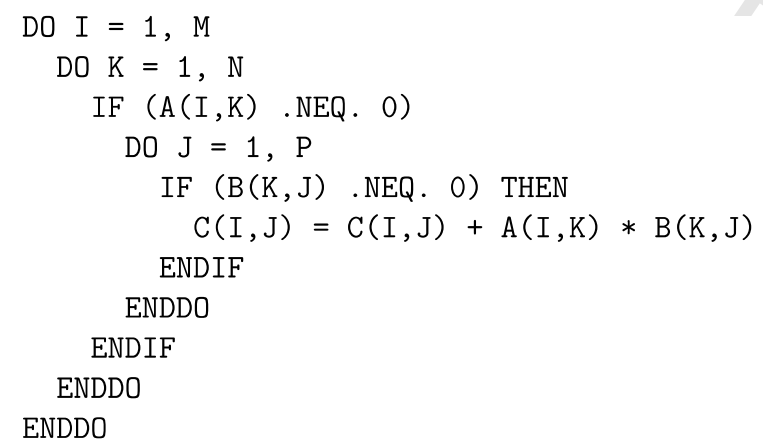

Fig. 5. Optimized product of matrices.

a conditional sentence that affects three of the references. Finally, Fig. 5 is an optimized product of matrices that contains references inside several nested conditional sentences. These conditionals try to avoid unuseful computations when one of their inputs is a zero.

In order to illustrate in detail our modeling strategy, we will explain step by step the modeling of the matrix product code, which is the most complex one. Then, the formulas for the references that experience non-regular access patterns in the other two codes will be provided for the sake of complexness. Finally, we will discuss the validation results.

\subsection{Optimized product modeling}

The code in Fig. 5 implements the product of two matrices, $\mathrm{A}$ and $\mathrm{B}$, which may have many zero entries. As an optimization, when the element of $A$ to be used in the current product is 0 , then all its products with the corresponding elements of $B$ are not performed. As an additional optimization, if the element of B to be used in the current product is 0 then that operation is not performed either. This avoids two floating point operations and the load and storage of $\mathrm{C}(\mathrm{I}, \mathrm{J})$.

Without loss of generality, we assume a compiler that maps scalar variables to registers and which tries to reuse the memory values recently read in processor registers. Under these conditions, the code in Fig. 5 contains three references to memory. The model in [7] can estimate the behavior of the reference $A(I, K)$, which takes place in every iteration of its enclosing loops. This, way we will focus our explanation on the modeling of the behavior of the references $C(I, J)$ and $B(K, J)$, since the access to $A(I, K)$ is not conditional, and thus it is already covered in previous publications.

\subsubsection{Modeling of $C(I, J)$}

The analysis of the behavior of this reference, 817 which we will call $R$ along this explanation for simplicity, begins in the innermost loop, in level two. In this level the loop variable indexes one of the references of one of the conditions that control the accesses of $\mathrm{C}(I, J)$, so the PME for this loop will be Eq. (3). As for its parameters, since $S_{R 2}=P$, then $L_{R 2}=1+N$ and $G_{R 2} \simeq 1$; and $p_{2}$ is the component in vector $\vec{p}$ associated to the probability that the condition inside the loop in nesting level 3 holds. Also, when expanding Eq. (4) we must take into account that this loop is in the innermost level, thus $F_{3}(R, \operatorname{RegIn}, \vec{p})=A V_{0}($ RegIn $)$. After the simplification the formulation is

$F_{2}(R, \operatorname{RegIn}, \vec{p})=p_{2} P A V_{0}(\operatorname{RegIn})$.

In the next upper level, level one, the loop variable indexes also one reference of one of the conditions, so the same equations are to be applied. In this loop, $S_{R 1}=0, L_{R 1}=1$ and $G_{R 1}=N$, so $F_{1}(R, \operatorname{RegIn}, \vec{p})=p_{1} \sum_{j=1}^{N} \mathrm{WMR}_{1}(R, \operatorname{RegIn}, j, \vec{p})$.

In order to compute $\mathrm{WMR}_{1}$ we need to calculate the 839 value for two functions. One is $P_{1}(R, \vec{p})$, which for 840 
841 our reference takes the value $p_{1} p_{2}$, where $p_{i}$ is the $i$ th 842 element in vector $\vec{p}$. The other one is $\operatorname{Reg}_{1}(R, i)$, the 843 region accessed during $i$ iterations of loop 1 that can 844 interfere with the accesses of our reference:

$$
\begin{aligned}
\operatorname{Reg}_{1}(R, i)= & R_{\mathrm{rp}_{\mathrm{self}}}\left(P, 1, M, 1-\left(1-p_{1} p_{2}\right)^{i}\right) \\
& \cup R_{\mathrm{r}}(i, 1, M) \cup R_{\mathrm{rp}}\left(P, i, N, 1-\left(1-p_{1} p_{2}\right)^{i}\right) .
\end{aligned}
$$

847 The first term is associated to the self-interference of 848 the reference we are studying. It is associated to the 849 access to $P$ groups of one element with stride $M$ and 850 every access takes place with a given probability. 851 This access pattern was analyzed in Section 3.1.2, 852 where the calculation of its cross-interference area 853 vector was explained in detail. The self-interference 854 area vector, which would be the one to apply in this 855 equation, follows similar steps. The second term, $856 R_{\mathrm{r}}(i, 1, M)$, represents the access to $i$ groups of 1 ele857 ment separated by a distance $M$. The last term rep858 resents the access to $P$ groups of $i$ elements 859 separated by a constant stride $N$, each individual 860 access taking place with a given probability $1-$ $861\left(1-p_{1} p_{2}\right)^{i}$. Here the cross-interference area vector 862 is used, so the explanation in Section 3.1.2 applies.

863 In the outermost level, the loop variable indexes 864 a reference used in one of the conditions. As a 865 result, Eq. (3) is to be applied again. In this case, $866 S_{R 0}=1, L_{R 0}=1+\left\lfloor(M-1) / L_{\mathrm{s}}\right\rfloor$ and $G_{R 0} \simeq L_{\mathrm{s}}$, so 867 the formulation is

$$
\begin{aligned}
F_{0}(R, \operatorname{RegIn}, \vec{p})= & \left(1+\left\lfloor(M-1) / L_{\mathrm{s}}\right\rfloor\right) \\
& \times \sum_{j=1}^{L_{\mathrm{s}}} \mathrm{WMR}_{0}(R, \operatorname{RegIn}, 0, j, \vec{p}) .
\end{aligned}
$$

As before, two functions must be evaluated to compute $\mathrm{WMR}_{0}$. They are $P_{0}(R, \vec{p})=1-(1-$ $\left.p_{1} p_{2}\right)^{M}$ and $\operatorname{Reg}_{0}(R, i)$, given by

$$
\begin{aligned}
\operatorname{Reg}_{0}(R, i)= & R_{\mathrm{rp}_{\text {self }}}\left(P, 1, M, 1-\left(1-p_{1} p_{2}\right)^{N}\right) \\
& \cup R_{\mathrm{r}}(N, i, M) \cup R_{l}\left(P N, 1-\left(1-p_{1}\right)^{L_{\mathrm{s}}}\right) .
\end{aligned}
$$

875 The first term is associated to the self-interference of 876 our reference, which is the access to $P$ groups of one 877 element separated by a difference $M$ and every ac878 cess takes place with a given probability. The second 879 term represents the access to $N$ groups of $i$ elements 880 separated by a distance $M$. The last element repre881 sents the access to $P N$ consecutive elements with a 882 given probability.
4.1.2. Modeling of $B(K, J)$

883

The innermost loop for this reference, which we 884 will now call $R$ along this section, is also the one in level 2. The variable that controls this loop, $\mathrm{J}$, is not used in the indexing of referenced found in conditions that control the execution of this reference, thus Eq. (1) is to be applied. As this is the innermost loop, in the evaluation of this equation, $F_{3}(R, \operatorname{RegIn}, \vec{p})=A V_{0} \operatorname{RegIn}$. Since $S_{R 2}=N$ and $L_{R 2}=P$, the formulation for this nesting level is $F_{2}(R, S(\operatorname{RegIn}), \vec{p})=P A V_{0}(\operatorname{Reg} I n)$.

The next level is level one. In this level the variable of the loops indexes references in the two conditional statements than affect our reference, so Eq. (3) applies again. In this case, $S_{R 1}=1, L_{R 1}=$ $1+\left\lfloor(N-1) / L_{\mathrm{s}}\right\rfloor$ and $G_{R 1} \simeq L_{\mathrm{s}}$, so the formulation is

$$
\begin{aligned}
F_{1}(R, \operatorname{RegIn}, \vec{p})= & p_{1}\left(1+\left\lfloor(N-1) / L_{\mathrm{s}}\right\rfloor\right) \\
& \times \sum_{j=1}^{L_{\mathrm{s}}} \mathrm{WMR}_{1}(R, \operatorname{RegIn}, j, \vec{p}) .
\end{aligned}
$$

We need to know $P_{1}(R, \vec{p})=p_{1}$ and the value of 903 the accessed regions $\operatorname{Reg}_{1}(R, i)$ to compute $\mathrm{WMR}_{1}: 904$

$$
\begin{aligned}
\operatorname{Reg}_{1}(R, i)= & R_{\mathrm{r}_{\mathrm{self}}}(P, 1, N) \cup R_{\mathrm{r}}(i, 1, M) \\
& \cup R_{\mathrm{r} p}\left(P, 1, M, p_{2}\right) .
\end{aligned}
$$

The first term is associated to the self-interference of 907 B, which is the access to $P$ groups of 1 elements sep- 908 arated with stride $N$. The second term represents the 909 access to A: $i$ groups of one element separated by a 910 distance $M$. The last element describes the access to 911 C: $P$ groups of one element separated by a distance 912 $M$, every access takes place with a given probability 913 $p_{2}$.

In the outermost level, the variable of the loop 915 indexes a reference in one of the conditions, so we 916 have to apply again Eq. (3). For this loop and refer- 917 ence, $S_{R 0}=0, L_{R 0}=1$ and $G_{R 0}=M$, so the formu- 918 lation is

$F_{0}(R, \operatorname{RegIn}, \vec{p})=\sum_{j=1}^{M} \mathrm{WMR}_{0}(R, \operatorname{RegIn}, j, \vec{p})$.

In this loop, $\mathrm{WMR}_{0}$ is a function of $P_{0}(R, \vec{p})=$ $1-\left(1-p_{1}\right)^{L_{\mathrm{s}}}$ and the value of the accessed regions $\operatorname{Reg}_{0}(R, i)$ :

$\operatorname{Reg}_{0}(R, i)=R_{l_{\mathrm{self}}}\left(P N, 1-\left(1-p_{1}\right)^{L_{\mathrm{s}}}\right) \cup R_{\mathrm{r}}(N, i, M)$ $\cup R_{\mathrm{r} p}\left(P, i, M, 1-\left(1-p_{1} p_{2}\right)^{N}\right)$. 
927 The first term is associated to the self-interference of 928 our reference, which is the access to $P N$ elements 929 with a given probability. The second term represents 930 the access to $N$ groups of $i$ elements separated by a 931 distance $M$. The last element represents the access to $932 P$ groups of $i$ elements separated by a distance $M$, 933 every access takes place with a given probability.

934 4.2. PMEs for the irregular accesses

935 in the synthetic benchmark

936 In the synthetic benchmark in Fig. 3 the only refer937 ence that generates an irregular access pattern is $938 \mathrm{C}(\mathrm{J})$, and it is due to the enclosing IF structure that 939 depends on a condition on $\mathrm{B}(\mathrm{J})$. The PME that 940 reflects the behavior of $\mathrm{C}(\mathrm{J})$ in the innermost loop is $F_{1}(R, \operatorname{RegIn}, \vec{p})=p_{1} N / L_{\mathrm{s}} \sum_{j=1}^{L_{\mathrm{s}}} \mathrm{WMR}_{1}(R, \operatorname{RegIn}, j, \vec{p})$,

943 substituting $L_{R i}=N / L_{\mathrm{s}}$ and $G_{R i}=L_{\mathrm{s}}$ for $i=1$ in 944 Eq. (3). In the calculation of $\operatorname{WMR}_{1}(R, \operatorname{RegIn}$, $945 j, \vec{p})$ in Eq. (4) we would use $P_{1}(R, \vec{p})=p_{1}$ and $946 \operatorname{Reg}_{1}(R, i)=R_{\mathrm{s}}(i) \cup R_{\mathrm{l}_{\mathrm{self}}}\left(i, 1-\left(1-p_{1}\right)^{\min (i, l s)}\right)$.

947 The PME associated to the behavior of $\mathrm{C}(\mathrm{J})$ in 948 the outermost loop, which provides the prediction 949 for the whole nest for this reference, is

$$
\begin{aligned}
F_{0}(R, \operatorname{RegIn})= & F_{1}(R, \operatorname{RegIn}) \\
& +(M-1) F_{1}\left(R, \operatorname{Reg}_{0}(R, 1)\right),
\end{aligned}
$$

952 substituting $N_{i}=M$ and $L_{R i}=1$ for $i=0$ in Eq. (1). 953 In this $\mathrm{PME}, \operatorname{Reg}_{0}(R, 1)=R_{\mathrm{s}}(1) \cup R_{\mathrm{s}}(N) \cup R_{\mathrm{self}}$ $954\left(N, 1-\left(1-p_{1}\right)^{L_{\mathrm{s}}}\right)$.

955 4.3. PMEs for the irregular accesses in the CRS

956 benchmark

957 The references that generate irregular accesses in 958 the CRS storage algorithm depicted in Fig. 4 are $959 \mathrm{~B}(\mathrm{posB})$ and $j \mathrm{~B}(\mathrm{posB})$, which are controlled 960 by a condition on $A(I, J)$. Both references follow 961 exactly the same irregular access pattern, so we only 962 provide here the formulas for the modeling of $963 \mathrm{~B}(\mathrm{p} \circ \mathrm{sB})$, as those of $j \mathrm{~B}(\mathrm{p} \circ \mathrm{sB})$ are analogous. 964 Starting the analysis in the innermost loop, we get $F_{1}(R, \operatorname{RegIn}, \vec{p})=p M / L_{\mathrm{s}} \sum_{j=1}^{L_{\mathrm{s}}} \mathrm{WMR}_{1}(R, \operatorname{RegIn}, j, \vec{p})$,

967 substituting $p_{i}=p, L_{R i}=M / L_{\mathrm{s}}$ and $G_{R i}=L_{\mathrm{s}}$ for $968 i=1$ in Eq. (3). In the calculation of $\mathrm{WMR}_{1}$
$(R, \operatorname{RegIn}, j, \vec{p})$ in Eq. (4) we would use $P_{1}(R, \vec{p})=969$

1 and $\operatorname{Reg}_{1}(R, i)=R_{\mathrm{s}}(i) \cup R_{\mathrm{s}}(i p)$. $\quad 970$

Finally, in the outermost loop, the number of 971 misses can be predicted as

972

$F_{0}(R, \operatorname{RegIn})=N F_{1}(R, \operatorname{RegIn})$,

substituting $N_{i}=N$ and $L_{R i}=N$ for $i=0$ in Eq. (1). 975

\subsection{Validation results}

In order to validate our model its predictions were compared with the results of trace drive simulations using different cache configurations, problem sizes and probabilities for the fulfillment of the conditionals for the three example codes. The combinations used to validate the model for each code are shown in Table 1 . Rows $M, N$ and $P$ correspond to the problem size, this is, the number of iterations of each loop, expressed as the value of its upper limit. Then come the probabilities $p_{i}$ that the conditional sentences found in the codes are true. The synthetic and the CRS codes have a single conditional and no $P$ loop, thus rows $P$ and $p_{2}$ are empty for them. Then, the cache configurations used in the validation are shown in the format $\left(C_{\mathrm{s}}-\right.$ $\left.L_{\mathrm{s}}-K\right)$, this is (cache size-line size-associativity). The cache and line sizes are expressed in words or elements of the matrices accessed, not in bytes. Then, Table 1 shows the total number of parameter combinations tried for each code taking into account the previous rows. For each one of these combinations a total of twenty five different simulations were made using different base addresses for the data structures. This improves the validation of the model by taking into account many different relative positions for the mapping on the cache of the different data structures. The last two rows in the table show the average and the maximum value for each code of the metric $\Delta_{\mathrm{MR}}$ that we use to measure the accuracy of the model. This metric is the average of the absolute value of the difference between the predicted and the measured miss rate (MR) in each one of the 25 simulations performed for each parameter combination. As expected, the average and maximum errors grow with the complexity of the code. Still, we consider that a maximum absolute error of only about $11 \%$ is very satisfactory. Also, the large difference between the average and the maximum $\Delta_{\mathrm{MR}}$ shows that (relatively) large errors are very infrequent and, in general, the predictions estimate well the cache behavior.
977 978 979 980 981 982 983 984 985 986 987 988 989 990 991 992 993 994 995 996 997 998 999 1000 1001 1002 1003 1004 1005 1006 1007 1008 1009 1010 1011 1012 1013 1014 1015 $\varsigma \varsigma$ 1017 1018 
Table 1

Parameter combinations used for the validation and average and maximum miss rate prediction error

\begin{tabular}{llll}
\hline Parameter & Kernel & & CRS \\
\cline { 2 - 4 } & Synthetic & $1000,1200,1400,1600,1800$ & Matrix product \\
\hline$M$ & $950,1750,2000,4500,6000$ & $1250,1350,2450,2650,3000$ & $350,550,400,600$ \\
$P$ & $1200,2500,3000,4000,9500$ & - & $250,350,450,650$ \\
$p_{1}$ & - & $0.1,0.2,0.3,0.4,0.5$ & $600,700,750,800$ \\
$p_{2}$ & $0.1,0.2,0.3,0.4,0.5$ & - & $0.1,0.2,0.3,0.4$ \\
& - & $4 \mathrm{~K}-4-1$ & $0.1,0.2,0.3,0.4$ \\
Cache & $4 \mathrm{~K}-4-1$ & $4 \mathrm{~K}-4-2$ & $4 \mathrm{~K}-4-1$ \\
Configurations & $4 \mathrm{~K}-4-2$ & $8 \mathrm{~K}-4-1$ & $4 \mathrm{~K}-4-2$ \\
$\left(C_{\mathrm{s}}-L_{\mathrm{s}}-K\right)$ & $8 \mathrm{~K}-4-1$ & $8 \mathrm{~K}-4-2$ & - \\
Sizes in words & $8 \mathrm{~K}-4-2$ & $16 \mathrm{~K}-8-2$ & $8 \mathrm{~K}-4-2$ \\
Combinations & $16 \mathrm{~K}-8-2$ & 625 & $16 \mathrm{~K}-8-2$ \\
Avg $\Delta_{\mathrm{MR}}$ & 625 & $1.43 \%$ & 4096 \\
Max $\Delta_{\mathrm{MR}}$ & $0.22 \%$ & $8.05 \%$ & $2.23 \%$ \\
\hline
\end{tabular}

Tables 2-4 show the validation results for some 1020 randomly chosen combinations of the problem size, 1021 the conditional probabilities and the cache configu1022 rations for the three codes proposed in Figs. 3-5, respectively. The columns in the three tables have 1023 the same meaning as the respective rows in Table 1024 1. Many of the combinations chosen in these tables 1025 do not belong to the set of experiments described by 1026

Table 2

Validation data for the synthetic kernel in Fig. 3 for several cache configurations, problem sizes and condition probabilities

\begin{tabular}{rlllrllrlr}
\hline$M$ & $N$ & $p$ & $C_{\mathrm{s}}$ & $L_{\mathrm{s}}$ & $K$ & $\Delta_{\mathrm{MR}}$ & $T_{\text {sim }}$ & $T_{\text {exe }}$ & $T_{\text {mod }}$ \\
\hline 50,000 & 47,500 & 0.4 & $16 \mathrm{~K}$ & 8 & 2 & 0.015 & 182.211 & 68.022 & 0.005 \\
50,000 & 47,500 & 0.2 & $8 \mathrm{~K}$ & 32 & 4 & 0.004 & 138.187 & 50.003 & 0.005 \\
22,000 & 14,500 & 0.4 & $32 \mathrm{~K}$ & 16 & 4 & 0.001 & 28.244 & 7.033 & 0.003 \\
22,000 & 14,500 & 0.4 & $8 \mathrm{~K}$ & 8 & 1 & 0.067 & 65.002 & 7.129 & 0.004 \\
18,000 & 22,000 & 0.2 & $32 \mathrm{~K}$ & 16 & 2 & 0.574 & 23.021 & 7.586 & 0.004 \\
18,000 & 22,000 & 0.1 & $16 \mathrm{~K}$ & 8 & 2 & 0.076 & 22.112 & 6.012 & 0.004 \\
18,000 & 22,000 & 0.3 & $4 \mathrm{~K}$ & 32 & 4 & 0.141 & 95.223 & 8.010 & 0.004 \\
14,500 & 19,500 & 0.7 & $64 \mathrm{~K}$ & 8 & 8 & 0.000 & 32.224 & 7.697 & 0.005 \\
14,500 & 19,500 & 0.2 & $16 \mathrm{~K}$ & 4 & 2 & 0.252 & 20.269 & 5.331 & 0.005 \\
14,500 & 19,500 & 0.3 & $8 \mathrm{~K}$ & 4 & 1 & 0.124 & 20.901 & 6.465 & 0.004 \\
1750 & 1750 & 0.4 & $8 \mathrm{~K}$ & 4 & 8 & 0.000 & 1.123 & 1.000 & 0.003 \\
1750 & 1750 & 0.7 & $8 \mathrm{~K}$ & 8 & 4 & 0.000 & 0.988 & 0.322 & 0.003 \\
\hline
\end{tabular}

Table 3

Validation data for the CRS code in Fig. 4 for several cache configurations, problem sizes and condition probabilities

\begin{tabular}{rrllrllrrr}
\hline$M$ & $N$ & $p$ & $C_{\mathrm{s}}$ & $L_{\mathrm{s}}$ & $K$ & $\Delta_{\mathrm{MR}}$ & $T_{\text {sim }}$ & $T_{\text {exe }}$ & $T_{\text {mod }}$ \\
\hline 6200 & 10,150 & 0.4 & $32 \mathrm{~K}$ & 8 & 4 & 0.01 & 16.308 & 4.022 & 1.225 \\
4200 & 17,150 & 0.1 & $4 \mathrm{~K}$ & 4 & 2 & 0.04 & 14.797 & 6.401 & 0.246 \\
16,220 & 7200 & 0.2 & $16 \mathrm{~K}$ & 4 & 2 & 0.03 & 27.477 & 5.011 & 3.646 \\
6200 & 14,250 & 0.3 & $32 \mathrm{~K}$ & 8 & 4 & 0.00 & 21.089 & 5.891 & 1.221 \\
9200 & 14,250 & 0.1 & $4 \mathrm{~K}$ & 4 & 8 & 0.04 & 37.768 & 11.001 & 1.196 \\
1100 & 15,550 & 0.5 & $4 \mathrm{~K}$ & 4 & 8 & 0.02 & 2.724 & 1.668 & 0.021 \\
2900 & 17,250 & 0.3 & $32 \mathrm{~K}$ & 16 & 4 & 0.17 & 10.363 & 4.573 & 0.572 \\
8900 & 9250 & 0.1 & $64 \mathrm{~K}$ & 8 & 4 & 0.64 & 17.119 & 11.228 & 2.516 \\
4200 & 12,150 & 0.1 & $4 \mathrm{~K}$ & 4 & 2 & 0.04 & 9.364 & 3.880 & 0.246 \\
5000 & 15,000 & 0.3 & $32 \mathrm{~K}$ & 8 & 4 & 0.11 & 17.852 & 10.330 & 0.804 \\
7200 & 12,250 & 0.1 & $4 \mathrm{~K}$ & 4 & 8 & 0.04 & 18.224 & 9.646 & 0.721 \\
\hline
\end{tabular}


Table 4

Validation data for the optimized matrix product code in Fig. 5 for several cache configurations, problem sizes and condition probabilities

\begin{tabular}{|c|c|c|c|c|c|c|c|c|c|c|c|}
\hline$M$ & $N$ & $P$ & $p_{1}$ & $p_{2}$ & $C_{\mathrm{s}}$ & $L_{\mathrm{s}}$ & $K$ & $\Delta_{\mathrm{MR}}$ & $T_{\text {sim }}$ & $T_{\text {exe }}$ & $T_{\text {mod }}$ \\
\hline 750 & 750 & 1000 & 0.2 & 0.1 & $16 \mathrm{~K}$ & 8 & 8 & 0.79 & 24.444 & 11.233 & 0.203 \\
\hline 750 & 750 & 1000 & 0.8 & 0.3 & $16 \mathrm{~K}$ & 16 & 16 & 1.31 & 86.845 & 72.069 & 0.987 \\
\hline 900 & 850 & 900 & 0.9 & 0.1 & $64 \mathrm{~K}$ & 8 & 8 & 0.59 & 85.358 & 65.266 & 0.990 \\
\hline 900 & 950 & 1500 & 0.1 & 0.4 & $32 \mathrm{~K}$ & 8 & 4 & 6.62 & 31.768 & 16.201 & 0.511 \\
\hline 900 & 950 & 1500 & 0.8 & 0.3 & $16 \mathrm{~K}$ & 4 & 2 & 2.04 & 171.755 & 85.023 & 0.149 \\
\hline 1000 & 850 & 900 & 0.7 & 0.5 & $4 \mathrm{~K}$ & 8 & 2 & 3.13 & 110.328 & 108.211 & 0.139 \\
\hline 200 & 250 & 150 & 0.8 & 0.2 & $16 \mathrm{~K}$ & 4 & 2 & 0.48 & 0.764 & 0.550 & 1.034 \\
\hline 200 & 250 & 150 & 0.1 & 0.3 & $32 \mathrm{~K}$ & 8 & 4 & 5.91 & 0.134 & 0.112 & 0.301 \\
\hline 200 & 250 & 150 & 0.3 & 0.1 & $4 K$ & 4 & 8 & 1.45 & 0.406 & 0.323 & 0.030 \\
\hline 100 & 350 & 90 & 0.8 & 0.5 & $4 \mathrm{~K}$ & 4 & 8 & 0.14 & 0.500 & 0.201 & 0.031 \\
\hline 100 & 350 & 90 & 0.4 & 0.4 & $8 \mathrm{~K}$ & 8 & 4 & 0.40 & 0.218 & 0.122 & 0.586 \\
\hline 100 & 350 & 90 & 0.2 & 0.3 & $4 \mathrm{~K}$ & 8 & 2 & 0.05 & 0.104 & 0.101 & 0.309 \\
\hline
\end{tabular}

1027 Table 1, so that the behavior of the model can be 1028 analyzed for a wider scope of parameters. The last 1029 three columns in each table correspond, respec1030 tively, to the simulation time, execution time and 1031 modeling times expressed in seconds and measured 1032 in a Athlon 2400 processor-based system $1033(2086 \mathrm{GHz})$. As we see, modeling times are much 1034 shorter than trace-driven simulation times despite 1035 the fact hat we use a very fast and simple simulator. 1036 In fact, many times they are even faster than the 1037 native execution times. Furthermore, sometimes 1038 modeling times are several orders of magnitude 1039 shorter than trace-driven simulation and even exe1040 cution times. The modeling time does not include 1041 the time required to build the formulas for the 1042 example codes. This will be made automatically by a tool we are currently developing. According to 1043 our experience in [10], the overhead of such tool is 1044 negligible.

Figs. 6 and 7 show the evolution of both the number of misses and the miss rate measured and predicted for different cache configurations and probabilities of the conditionals for the CRS and the matrix product codes, respectively. The figures show, as the previous tables, that the model is successful in predicting the behavior of the cache. A new interesting conclusion we can draw from these figures is that our extended model is indeed required to predict correctly the behavior of the memory hierarchy when irregular access patterns are involved. We can see that a simplified model that did not support irregular access patterns and which
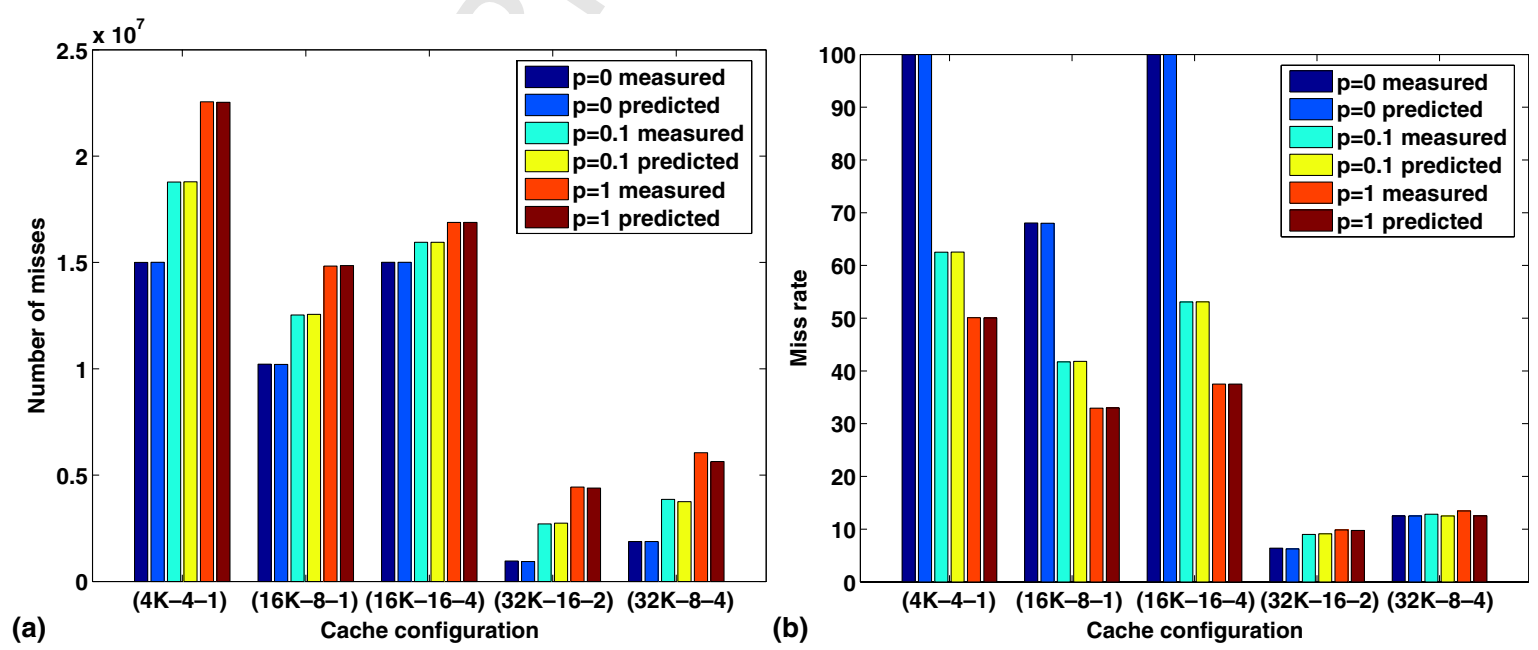

Fig. 6. Measured versus predicted (a) misses and (b) miss rates for several cache configurations and different probabilities of verification of the conditionals for the CRS code with $M=1500$ and $N=10,000$. The cache configurations are expressed as $\left(C_{\mathrm{s}}-L_{\mathrm{s}}-K\right)$, with sizes in matrix elements. 

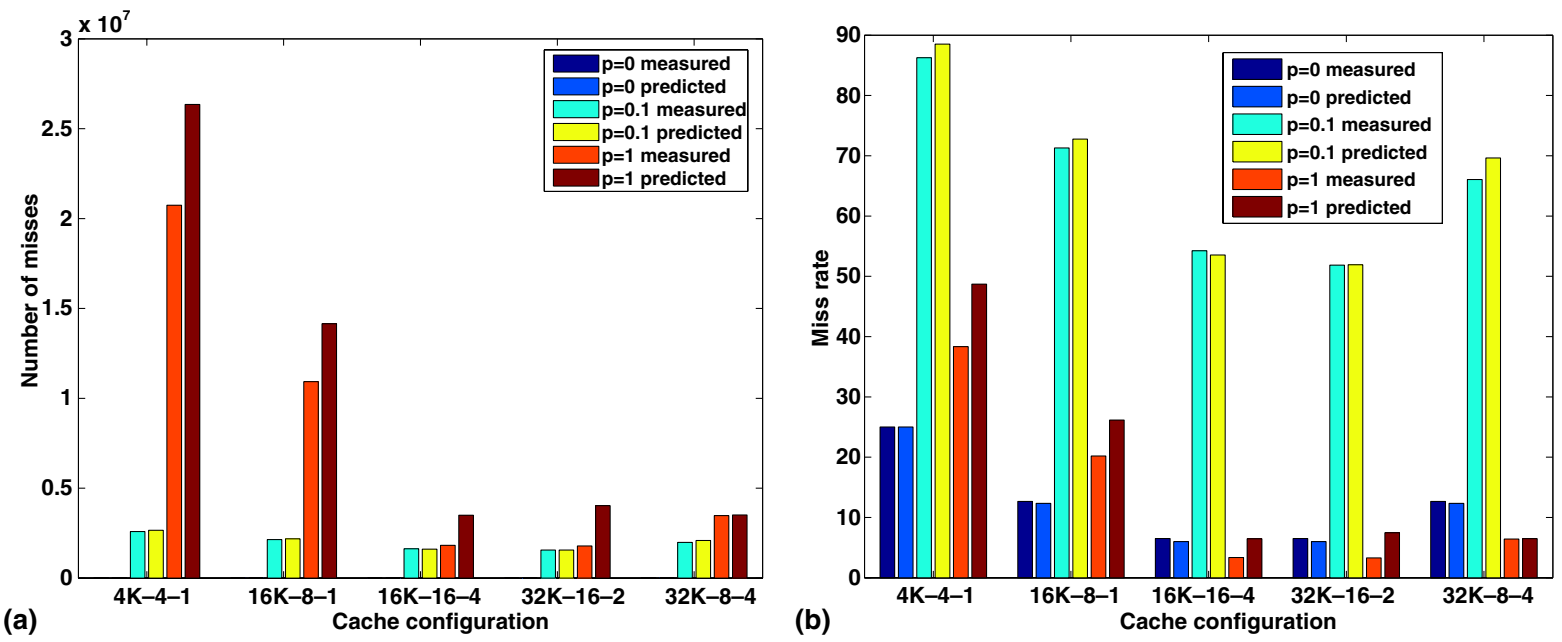

Fig. 7. Measured versus predicted (a) misses and (b) miss rates for several cache configurations and different probabilities of verification of the conditionals for the optimized matrix product code with $M=300, N=300$ and $P=300$. The cache configurations are expressed as $\left(C_{\mathrm{s}}-L_{\mathrm{s}}-K\right)$, with sizes in matrix elements.

1059 chose to make all probabilities either 0 or 1 (the two 1060 extremes cases) would yield predictions very differ1061 ent from the real values obtained for intermediate 1062 probabilities like 0.1 , shown in the figures. This jus1063 tifies the interest of our research.

\section{5. Related work}

1065 There are a number of previous works that also 1066 try to study and improve the behavior of the mem1067 ory hierarchy by means of analytical models based 1068 on the structure of the code. Among those works 1069 we find [11], which is restricted to the modeling of 1070 direct-mapped caches and that lacks an automatic 1071 implementation. Later [12,4], overcame some of 1072 these limitation. This way [12], is based on the con1073 struction of the cache miss equations (CMEs), 1074 which are lineal system of Diophantine equation, 1075 where each solution corresponds to a potential 1076 cache miss. One of its main limitations is its high 1077 computing cost. The computing times required by 1078 [4] are much shorter, and similar to those of our 1079 model, however, its errors are larger than those of 1080 our model. Both works share the limitation that 1081 their modeling is only applicable to regular access 1082 patterns found in perfectly nested loops, and they 1083 do not take into account the possible reuses in struc1084 tures that have been accessed in previous loops. This 1085 is a very important subject, as most misses in 1086 numerical codes are inter-nest misses [13], which implies that optimizations should consider several 1087 nests. 1088

More recently [5,6], allow the analysis of non- 1089 perfectly nested loops and consider the reuse 1090 between loops in different nests. The former is based 1091 on Presburger formulas and provides very accurate 1092 estimations for small kernels but it can only handle 1093 modest levels of associativity (for example its vali- 1094 dation only considers degrees of associativity one 1095 and two), and it is very time-consuming, which 1096 reduces its applicability. In fact, running a simula- 1097 tion is much faster than solving the equations this 1098 model generates. As for the latter, it is based on 1099 the extension of [14] in order to quantify the reuse, 1100 and it applies the CMEs of [12] in order to estimate 1101 the number of misses. The time it requires to solve 1102 the CMEs is reduced considerably by applying sta- 1103 tistical techniques that allow to provide a prediction 1104 within a confidence interval. This model can analyze 1105 complete programs, imposing the conditions that 1106 the accesses follow regular patterns and that the 1107 codes do not contain data-dependent constructions, 1108 neither in the loop conditions nor in the conditional 1109 sentences. The model precision is similar to that of 1110 ours in most of the cases, however its computing 1111 times are longer. In a later work [9], this model 1112 was extended to consider continual sentences that 1113 could be analyzed statically at compile-time and 1114 were based on the indexes of the loops, not on the 1115 data read or computed in the program. These condi- 1116 
1117 tionals follow predictable and mostly regular access

1118 patterns, so there is little relation to our work.

1119 Unlike our model, all these approaches require

1120 knowing the base addresses of the data structures.

1121 This restricts their scope of application, as these

1122 addresses are not available in many situations

1123 (physically-addressed caches, dynamically allocated

1124 data structures,...). Besides, none of them can

1125 model codes with data-dependent conditions.

1126 Indeed, it is the probabilistic nature of our model

1127 what allows us to consider this broad scope of

1128 codes.

\section{Conclusions}

1130 In this work we have presented an extension to 1131 the PME model described in [7]. The extension 1132 allows this model to be the first one that can analyze 1133 codes with data-dependent conditionals. The 1134 extended model can handle conditionals nested in 1135 any arbitrary way that can affect isolated references 1136 or whole loop nests. We are currently limited by the 1137 fact that the conditions must follow an uniform dis1138 tribution, but we think our research is an important 1139 step in the direction of broadening the scope of 1140 applicability of analytical models. This raises the 1141 possibility of driving compiler optimizations for 1142 codes with irregular access patterns based on com1143 pile-time estimations of the model, and helps under1144 stand better the complex behavior of these codes. 1145 Our experiments show that the model provides 1146 accurate estimations of the number of misses 1147 generated by a given code while requiring quite 1148 short computing times. Typical prediction errors 1149 and within $2 \%$ of the miss rate, and maximum 1150 errors, which are quite infrequent, range between $11513.8 \%$ and $11.3 \%$ depending on the complexity of 1152 the code.

1153 We are now working in an extension of our 1154 model to consider non-uniform distributions of 1155 probability for the accesses. We are also developing 1156 an automatic implementation of the extension of the 1157 model described in this paper in order to integrate it 1158 in a compiler framework, in a similar way to what 1159 was done with the original model [10]. We plan to 1160 use the Polaris [15] compiler framework as platform 1161 for this purpose, although the model can be coupled 1162 with any other front-end and used to model any 1163 programming language. As for the scope of the pro1164 gram structures that we wish to be amenable to 1165 analysis using the PME model, our next step will be to consider codes with irregular accesses due to 1166 the use of indirections or pointers.

\section{References}

[1] R. Uhlig, T. Mudge, Trace driven memory simulation: a survey, ACM Computing Surveys 29 (2) (1997) 128-170.

1169

1170

[2] M. Zagha, B. Larson, S. Turner, M. Itzkowitz, Performance 1171 analysis using the MIPS R10000 performance counters, in: 1172 ACM (Ed.), Proc. Supercomputing '96 Conference, ACM 1173 Press and IEEE Computer Society Press, 1996, pp. 17-22.

[3] S. Ghosh, M. Martonosi, S. Malik, Cache miss equations: an analytical representation of cache misses, in: Proc. 11th ACM Int'l. Conf. on Supercomputing (ICS'97), ACM Press, 1997, pp. 317-324.

[4] J.S. Harper, D.J. Kerbyson, G.R. Nudd, Analytical modeling of set-associative cache behavior, IEEE Transactions on Computers 48 (10) (1999) 1009-1024.

[5] S. Chatterjee, E. Parker, P. Hanlon, A. Lebeck, Exact analysis of the cache behavior of nested loops, in: Proc. of the ACM SIGPLAN'01 Conference on Programming Language Design and Implementation (PLDI'01), 2001, pp. 286297.

[6] X. Vera, J. Xue, Let's study whole-program behaviour analytically, in: Proc. of the 8th Int'l Symposium on HighPerformance Computer Architecture (HPCA8), 2002, pp. 175-186.

[7] B.B. Fraguela, R. Doallo, E.L. Zapata, Probabilistic miss equations: evaluating memory hierarchy performance, IEEE Transactions on Computers 52 (3) (2003) 321-336.

[8] Y. Paek, J. Hoeflinger, D. Padua, Simplification of array access patterns for compiler optimizations, ACM SIGPLAN Notices 33 (5) (1998) 60-71.

[9] X. Vera, J. Xue, Efficient compile-time analysis of cache behaviour for programs with IF statements, in: International Conference on Algorithms and Architectures for Parallel Processing, 2002, pp. 396-407.

[10] B.B. Fraguela, J. Tourino, R. Doallo, E.L. Zapata, A compiler tool to predict memory hierarchy performance of scientific codes, Parallel Computing 30 (2) (2004) 225248.

[11] O. Temam, C. Fricker, W. Jalby, Cache interference phenomena, in: Proc. Sigmetrics Conference on Measurement and Modeling of Computer Systems, ACM Press, 1994, pp. 261-271.

[12] S. Ghosh, M. Martonosi, S. Malik, Cache miss equations: a compiler framework for analyzing and tuning memory behavior, ACM Transactions on Programming Languages and Systems 21 (4) (1999) 702-745.

[13] K.S. McKinley, O. Temam, Quantifying loop nest locality using SPEC'95 and the perfect benchmarks, ACM Transactions on Computer Systems 17 (4) (1999) 288-336.

[14] M.E. Wolf, M.S. Lam, A data locality optimizing algorithm, in: Proc. of the ACM SIGPLAN '91 Conference on Programming Language Design and Implementation, 1991, pp. $30-44$.

[15] W. Blume, R. Doallo, R. Eigenmann, J. Grout, J. Hoeflinger, T. Lawrence, J. Lee, D. Padua, Y. Paek, B. Pottenger, L. Rauchwerger, P. Tu, Parallel programming with Polaris, IEEE Computer 29 (12) (1996) 78-82. 


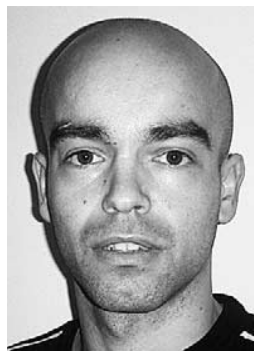

Diego Andrade received the MS degree in computer science from the University of A Coruna, Spain, in 2002. He is currently a PhD student in the Department of Electronics and Systems in the University of A Coruna. His research interests are about evaluation and prediction, analytical modeling, high performance simulation and compiler transformations.

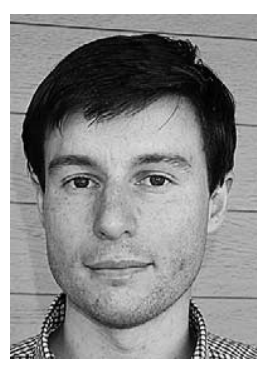

Basilio B. Fraguela received both the MS degree and the $\mathrm{PhD}$ degree in computer science from the University of A Coruna, Spain, in 1994 and 1999, respectively. He is currently an associate professor in the Department of Electronics and Systems of the University of A Coruna. His primary research interests are in the fields of performance evaluation and prediction, analytical modeling, high performance simulation and compiler transformations.

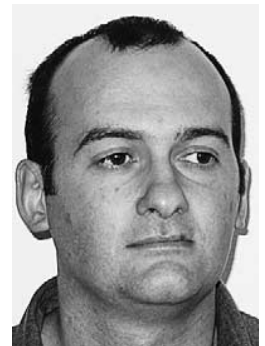

Ramon Doallo received his BS and MS degrees in Physics from the University of Santiago de Compostela in 1987, and his $\mathrm{PhD}$ in Physics from the University of Santiago de Compostela in 1992. In 1990 he joined as associate professor the Department of Electronics and Systems of the University of A Coruna, Spain, where he became full professor in 1999. He has extensively published in the areas of computer architecture, and parallel and distributed computing. He is member of the IEEE Computer Society. 